\title{
An Approach towards Design of N-Bit AES to Enhance Security using Reversible Logic
}

\author{
Rohini Hongal \\ ECE, Dept \\ KLE, Technological University \\ India
}

\author{
Jyoti H \\ ECE, Dept \\ KLE, Technological University \\ India
}

\author{
Rajashekar S \\ ECE, Dept \\ KLE, Technological University \\ India
}

\begin{abstract}
The main aim of this paper is to provide higher security for several applications over the Internet by enhancing the overall strength of the existing Advanced Encryption Standard algorithm (AES). The standard AES uses block size of 128-bit and key sizes of 128, 192, 256 bits. AES is the most popular ,highly secure, faster and strong symmetric key, block cipher cryptographic algorithm today. But in nowadays, cyberattacks are continuously developing. Shannon's theorem states that a one-on-one relationship between each message bit to each key bit (hence both key and message length equal) would give the best security. To improve the security of the original AES, message size is increased from standard block size of 128 bits to $192,256,512$ bits and key size is made as equal as the message block size. We get more security by the use of larger key size and the increased throughput from the larger input block size compared to original AES of 128 bits. The implementation of proposed algorithm require more area but it can be accepted as most of the applications require high level of security and high throughput. Further, paper discusses the use of reversible logic to mitigate the power attacks in conventional AES as reversible gates offer ideally zero internal power dissipation. The proposed work is designed and implemented using Xilinx tool and verified on Virtex-5 FPGA using chipscope. Results are discussed w.r.t power, delay and hardware required in terms of gate count is calculated and implemented using only Feynman gate with quantum cost 1 .
\end{abstract}

\section{General Terms}

Security, Algorithms.

\section{Keywords}

Chipscope, FPGA, Reversible gates, Symmetric key, Quantum Cost.

\section{INTRODUCTION}

There were several hardware implementations of AES algorithm with standard block size of 128 and standard key size of 128,192,256 bits, as in [1].These various implementations of AES depend on the applications. Some applications need higher level of security with area restriction, where as some applications need higher throughput and stronger security without caring about area or time limitations. The various AES algorithms can be implemented based on our requirements. Many hardware implementations for AES were found in literature. These implementations were done for the standard AES of 128 block size with 128,192,256 bits key size, such as the work proposed in [1][2] [3]. The paper [1] presents the hardware implementation of AES Rijndael. Both encryption and decryption of AES algorithm are implemented using Xilinx Virtex-7 FPGA. The pre-calculated look-up tables (LUT) are used .High throughput and low latency are obtained in this approach. Less complex architecture is obtained by using pre-calculated LUTs. All the three standard key sizes of AES with standard block size of 128 are designed using Verilog - HDL. Advanced encryption standard AES-192 with multiple keys is proposed in the paper [2]. It is implemented using Field Programmable Gate Array (FPGA). The hardware implementation of AES algorithm with key size 128 bits and block size 128 bits is proposed in the paper[3].The algorithm is implemented using FPGA Artix7 Nexys 4 kit by configuring it with the help of Software Development Kit (SDK) in Xilinx ISE design suite. The paper [4] proposes the implementations of all the three standard key sizes of AES $(128,192,256)$ with block size of 128 which will use 10, 12 and 14 iterations respectively. In this approach, the cloud user is provided with all the three key sizes and based on the size ,cloud user will select one of the three encryption techniques and process is done.

The paper [5] explains AES algorithm with hybrid approach of Dynamic Key Generation and Dynamic S-box Generation. In this approach first more complexity is added in data to increase Confusion and Diffusion in Cipher text by using Dynamic Key Generation and then by using Dynamic S-Box Generation. Our proposed paper presents variation of the original AES algorithm with message and key size equal. As more security is needed for applications which need high level of security regardless of area and computational time but due to increase in message size and block size complexity of algorithm is increased. The proposed AES algorithm with equal key and message size provides high level of security and the increased throughput. Using equal key size as that of message results in more security, and more throughput from using two, four times larger block size(i.e. 256,512 block size) than the block size used in the original AES.-128[6]. Our proposed AES algorithm uses 192,256,512 block sizes and same key sizes as that of block size[7][8]. This new algorithm is designed and synthesized using Hardware Description Language (HDL).The reversible logic gates are used to implement the design[9][10].

\subsection{Reversible Logic Gates}

Reversible logic is widely used nowadays and has got more popularity due to their ability to reduce the power dissipation which is the main requirement in low power VLSI design. A reversible logic gate has an n-input and n-output .It is a logic device with one-to-one mapping which means we can determine the outputs from the inputs and vice versa. We can implement one or more applications using single reversible logic gate. 
Feynman gate is a $2 * 2$ one through reversible gate as shown in Fig1. The inputs are A, B and the outputs are $\mathrm{X}, \mathrm{Y}$ where $\mathrm{Y}=\mathrm{A}, \mathrm{X}=\mathrm{A} \oplus \mathrm{B}$. One of the outputs of Feynman gate is XOR gate operation and the other output is known as garbage which is used to maintain the reversibility [9][10].

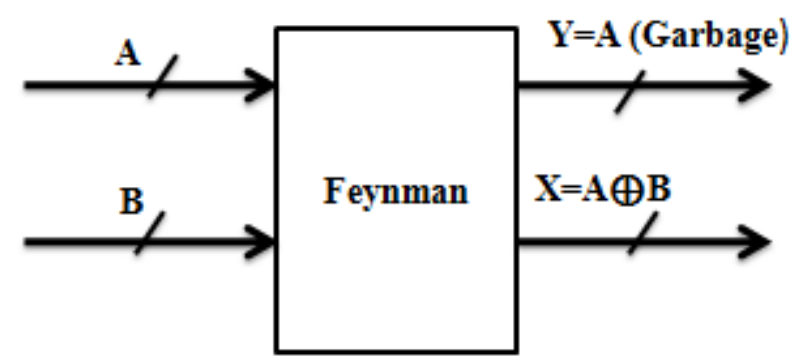

Fig 1: Feynman Gate

\section{IMPLEMENTATION DETAILS}

The new algorithm consist of the structure which is similar to original AES algorithm but having slight difference that is instead of using 128 bit plain text size and key size 128,192 or 256 bits, uses plaintexts of 192,256 and 512 bits and key size as that of message size 192,256 and 512 respectively that has impact on the whole algorithm structure. There are four transformations in the encryption of AES namely, Byte Substitution, Shift Row, Mix Column and Round Key Addition [11][12].

Analysis of Rijndael Algorithm passes through the 4 layers consists of

\subsection{Byte Substitution}

The AES algorithm performs operations on a two-dimensional array of bytes called the State. The state matrix consists of four rows of bytes, each containing $\mathrm{N}$ bytes, where $\mathrm{N}$ is the block length divided by 32 . Each byte of data in state matrix is replaced by byte of data from look up table.

\subsection{Shift Row Transformation}

In shift row transformation, the rows of the state matrix are cyclically shifted by its row number.

- The first row (Row 0) is not shifted.

- The Row 1 is cyclically shifted by 1 byte to the left.

- The Row 2 is cyclically shifted by 2 the left.

- The Row 3 is cyclically shifted by 1 byte to the left or 3 bytes to the right

The shift row transformation of 8 -bit data is shown in Fig 2.It is implemented using reversible logic gate Feynman. In Fig $2.2 \mathrm{~N}$ is the amount to be shifted .The same procedure is repeated $\mathrm{N}$ times. The same procedure is followed for 48,64 and 128 bit data transformation in case of $192,256,512$ respectively.

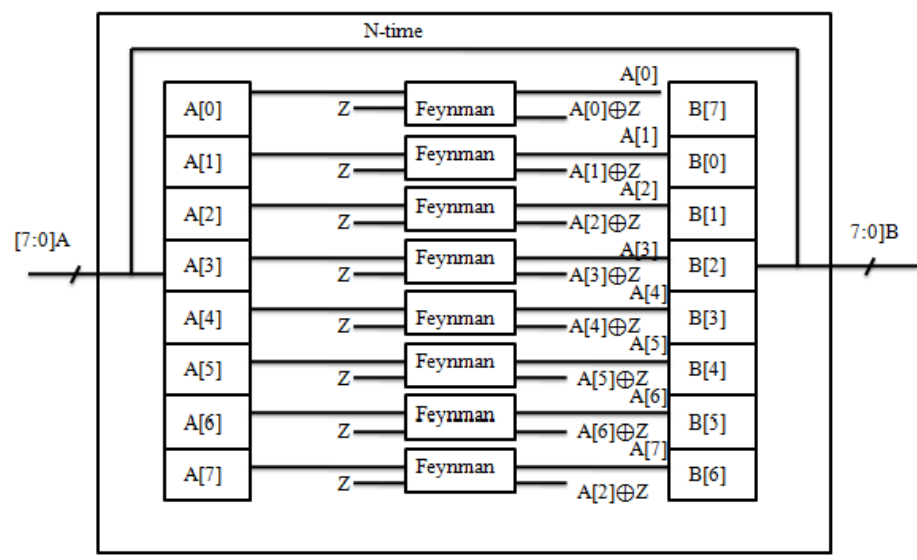

Fig 2: Reversible implementation of shift row transformation

\subsection{Mix Column Transformation}

The Mix column performs operations on the columns of the state matrix. The data is arranged in the form of two dimensional array of bytes known as state matrix. Each row of bytes in the state matrix is multiplied with the corresponding columns of constant matrix of $4 \mathrm{x} 4$.Mix column transformation is simulated using reversible logic gates. The fixed matrix used in our design is given below.

$\begin{array}{llll}02 & 03 & 01 & 01\end{array}$

$\begin{array}{llll}01 & 02 & 03 & 01\end{array}$

$\begin{array}{llll}01 & 01 & 02 & 03\end{array}$

$\begin{array}{llll}03 & 01 & 01 & 02\end{array}$

Multiplication of byte of data by 0 can be implemented using reversible gate shown in Fig 3.

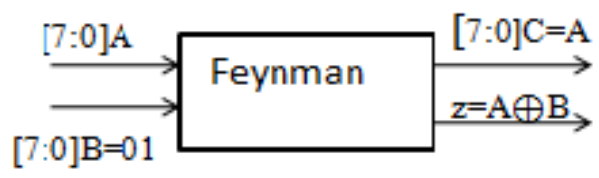

Fig 3: Reversible implementation of multiplication by constant value 01

Multiplication of byte of data by 02 can be implemented as shown in Fig 4. First byte of data is shifted left by 1-bit and then the shifted data is bitwise XOR ed with 00011011(1B) if the leftmost bit(LSB) of the original value before shift operation is 1. If MSB is 0, only 1-bit left shift is performed.

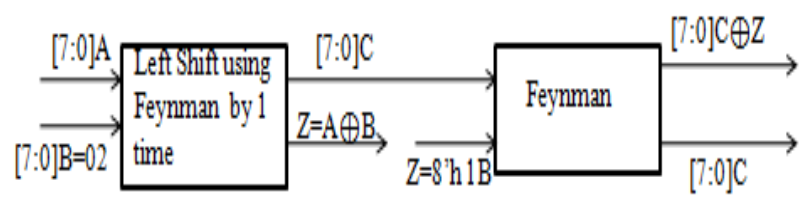

Fig 4: Reversible implementation of multiplication by constant value 02

Multiplication of byte of data by 03 can be implemented using reversible logic gate as shown in Fig 5 . The byte of data is multiplied by 2 and then conditional bitwise XOR with the original byte of data before shifting it. The same condition is applied for multiplication by 2 as explained above. 


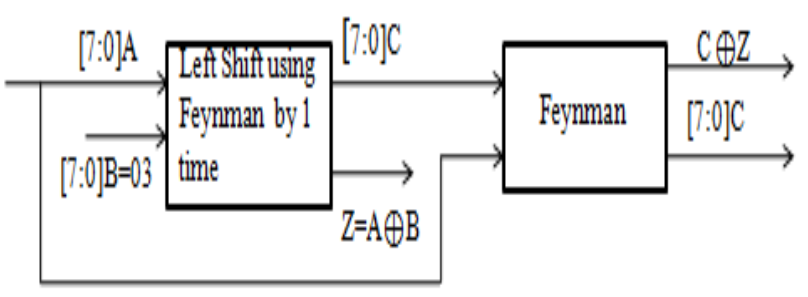

Fig 5: Reversible implementation of multiplication by constant value 03

The multiplication of $2 \times 2$ matrix can be written as

$$
\left[\begin{array}{ll}
A 1 & A 2 \\
A 3 & A 4
\end{array}\right]\left[\begin{array}{ll}
B 1 & B 3 \\
B 2 & B 4
\end{array}\right]=\left[\begin{array}{ll}
C 1 & C 2 \\
C 3 & C 4
\end{array}\right]
$$

The reversible implementation of $2 \times 2$ matrix is shown in Fig 6 . The multiply is carried out as explained above for different cases (i.e by 01,02,03). The same design is used for multiplication of $4 \times 6,4,8$ and $4 \times 16$ matrix in case of 192,356 and 512 bits data respectively.

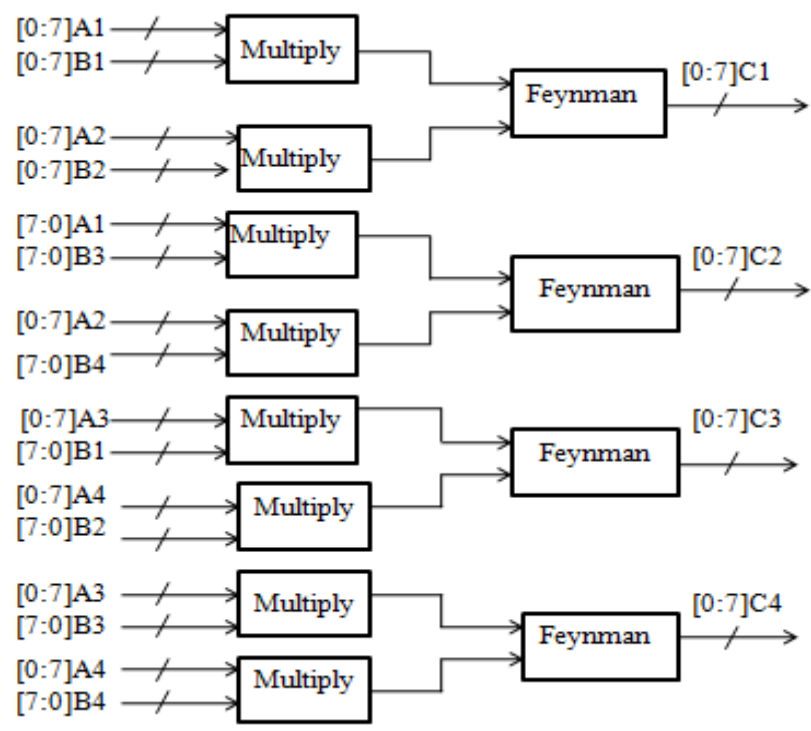

Fig 6: Reversible implementation of mix column matrix for $2 \times 2$ matrix

\subsection{Add Round Key}

There are different number of rounds depending on the key size and block size used. In the first round of encryption, the main key is XOR ed with the plaintext. In the remaining rounds except final round the sub keys obtained for different rounds are XOR ed with the output obtained from the mix column transformation. In the final round the last round sub key is XOR ed with the output of shift row transformation. Reversible logic of addround key is shown in Fig 7.

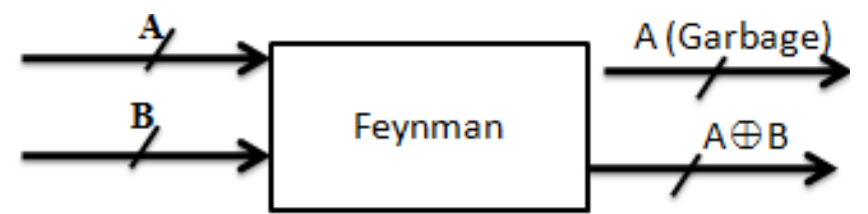

Fig 7: Reversible implementation of addround key

\subsection{Example of State Matrix Arrangement}

The arrangement of state matrix for all the three plaintexts (i.e, 192,256,512) can be written as.

1. AES encryption process takes input of 192-bit plaintext and 192 bit key and produces output of 192 - bit ciphertext. The 192-bit data blocks are divided by 32 to get number of bytes in each row. Dividing 192 by 32 we will get 6. So in each row 6 bytes and therefore there are four rows .So total number of bytes is $6 \times 424$ bytes. These 24 bytes are arranged as $4 \times 6$ State Matrix.

Plaintext in Hex(192 bits) : 4532 E6 EF 22 4A 67891942 A6 5D 5329 A6 2043922598 6A 4D 3F 2A

State Matrix can be
45221953436 A
32 4A 4229 A6 20
E6 67 A6 A6 25 3F
EF 89 5D 2098 2A

2. The 256-bit data blocks are divided by 32 to get number of bytes in each row. Dividing 256 by 32 we will get 8 .So in each row 8 bytes and therefore there are four rows .So total number of bytes is $8 \times 432$ bytes. These 32 bytes are arranged as $4 \times 8$ State Matrix.

Plaintext in Hex (256 bits): 55866734 A3 E0 8D 9745 4E 57 E8 79 0F 62574397 F6 2B FA 6E 5602 E4 A9 E6 15 2C $54379 \mathrm{~F}$

State Matrix can be written as

$$
\begin{array}{llllllll}
55 & \text { A3 } & 45 & 79 & 43 & \text { FA } & \text { E4 } & 2 \mathrm{C} \\
86 & \text { E0 } & 4 \mathrm{E} & 0 \mathrm{~F} & 97 & 6 \mathrm{E} & \text { A9 } & 54 \\
67 & 8 \mathrm{D} & 57 & 62 & \mathrm{~F} 6 & 56 & \mathrm{E} 6 & 37 \\
34 & 97 & \mathrm{E} 8 & 57 & 2 \mathrm{~B} & 02 & 15 & 9 \mathrm{~F}
\end{array}
$$

3.The 512-bit data blocks are divided into groups of 64 bytes each, and arranged as $4 \times 16$ State Matrix. The round key function is performed 11 times instead of 22 i.e, according to smith formula we will get 22 i.e, $\mathrm{Nb} / 4+6$, where $\mathrm{Nb}$ is the number of bytes of key size. As key size and message size is increased, the number of rounds has been reduced.

> Plaintext in Hex (512 bits): 01 C8 24 E1 3C F2 BC 18 E7 93 B5 BD 4729 A2 3E 63 AB 1F 4A EB 03 4E 59 9F AF $22 \mathrm{~B} 8 \mathrm{~A} 0 \quad \mathrm{C} 774 \mathrm{~A} 1 \mathrm{AB} 74$ 4E 7B 4849 8D 06 0E 8E D7 96 E5 1B 4078 5C 43 C6 A9 62 0E 57 C0 C8 0908 EB FE 3D F8 7F 37

State Matrix can be written as

$\begin{array}{llllllllllllll}01 & 3 \mathrm{C} & \mathrm{E} 7 & 47 & 63 & \mathrm{~EB} & 9 \mathrm{~F} & \mathrm{~A} 0 & \mathrm{AB} & 48 & 0 \mathrm{E} & \mathrm{E} 5 & 43 & 0 \mathrm{E}\end{array}$ 09 3D

$\begin{array}{llllllllllllll}\text { C8 } & \text { F2 } & 93 & 29 & \text { AB } & 03 & \text { AF } & \text { C7 } & 74 & 49 & 8 \mathrm{E} & 1 \mathrm{~B} & \mathrm{C} 6 & 57\end{array}$ 08 F8

$\begin{array}{llllllllllllll}24 & \text { BC } & \text { B5 } & \text { A2 } & 1 \mathrm{~F} & 4 \mathrm{E} & 22 & 74 & 4 \mathrm{E} & 8 \mathrm{D} & \mathrm{D} 7 & 40 & \mathrm{~A} 9 & \mathrm{C} 0\end{array}$ EB $7 \mathrm{~F}$

$\begin{array}{llllllllllllll}\mathrm{E} 1 & 18 & \mathrm{BD} & 3 \mathrm{E} & 4 \mathrm{~A} & 59 & \mathrm{~B} 8 & \mathrm{~A} 1 & 7 \mathrm{~B} & 06 & 96 & 78 & 62 & \mathrm{C} 8\end{array}$ FE 37 


\subsection{AES -256 Round 0 -14 Cipher Texts}

AES encryption process takes as input an 256-bit plaintext and 256 bit key and produces as output an 256 - bit ciphertext.

Plaintext:

54732067544F4E2068204B20776E6954616D75466F656E77 74796E752020656F

Key

4537027645F4E6820204B2077656954616789466F656E76D 4796E752020656F7

Round0:

1144221111bba8a26a24f9270138fc127715e1209933891a33e f892722263398

Round1:

6f8b40c6ef89ff040f594668c100aa0e6e5f08c9180f33a684fc5c c8c4d9e192

Round2:

d9704b26c76648c5bef0cb38ef9f2310eb2f0d98595f1d4f43aae e2231c256d1

Round3:

0284122aeba53e3c8438157c908859f0c837ae36f62ff78396c2 c2e $1 \mathrm{ad} 3 \mathrm{a} 4 \mathrm{c} 31$

Round4:

3f87b15cb0801100c05b4801054ec5b12b940243c2cfb90e36d 6d949bc4a6504

Round5:

aba332315e369f53fe8f19f613a15d81e1ec6998654ff77ddc9a5 c0eba19872e

Round6:

78bea8ef8f7fe5d86ca310ab016dae9ba6dfe08df84add82417e2 9de85ac3a67

Round7:

1721b141e0c68080837bca4eeae7f8535ce26870ac1720c49dd $925 \mathrm{f} 215 \mathrm{ec} 052 \mathrm{e}$

Round8:

5b931b9c76a240120d6cbd33d8e5d8330683361ce30669ef522 dbec2e8dcc316

Round9:

7e1008493cdabbf2036fa968d43979c882feb32143554eb9cf89 d62f739f 3141

Round10:

1ad5ac5b1b2d864cf76aef3864b2d50ccfcee720590334f143f1c $40 \mathrm{ef} 18 \mathrm{e} 3 \mathrm{e} 7 \mathrm{f}$

Round11:

9bd19bc92d700143ef0eaba042a77acdec74c556b2101359139

e9aa22324951c

Round12:

93371181e14b70cb105d82e8002542195a96ec3bd871f5b6c2f $0668167 \mathrm{cf} 4217$

Round13:

b20000f839cdc9495814f0280cb7010d3f27efb4a5228dc41b86 $66 f 15 \mathrm{a} 5 \mathrm{a} 851 \mathrm{~d}$

Round14:

163f41a97f7f215671179d677b0518a080377512626de422de1 $220742 \mathrm{c} 9 \mathrm{~b} 6 \mathrm{f} 18$

\section{RESULTS AND DISCUSSIONS}

Verilog coding is done to check functionality of proposed work and verified on Vrtex5 (ML505) FPGA using chipscope. Simulation results of all 3 key size AES (192bit, 256bit and 512bit) are shown in figures 8.9.10. respectively. Also using tool power and delay generated is tabulated in Table 1 and shown in Fig 11,12 \& 13. .Hardware required in different phases of design in terms gate count is tabulated in Table 2.

\subsection{Simulation Result of 192 Bit Plaintext} And Key

plaintext:

45221953436a324a4229a620e667a6a6253fef895d20982a key:

54732067544f68204b20776e616d75466f6574796e752020

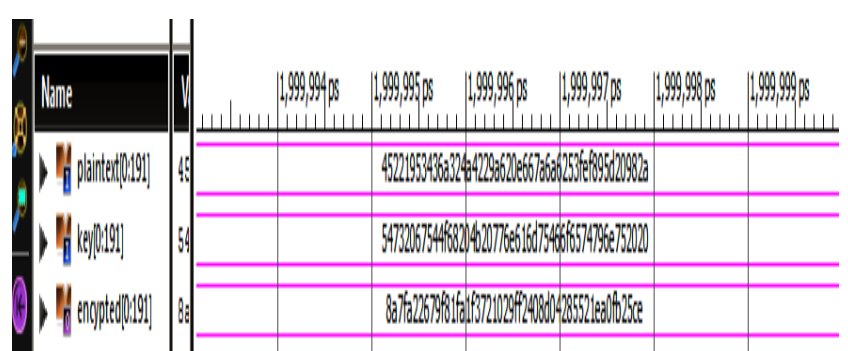

Fig 8: Simulation result of 192 bit

\subsection{Simulation Result of 256 Bit Plaintext}

\section{And Key}

Plaintext:

54732067544F4E2068204B20776E6954616D75466F656E77 $74796 \mathrm{E} 752020656 \mathrm{~F}$

Key:

4537027645F4E6820204B2077656954616789466F656E76D $4796 \mathrm{E} 752020656 \mathrm{~F} 7$

Encrypted Output:

163f41a97f7f215671179d677b0518a080377512626de422de1 220742c9b6f18

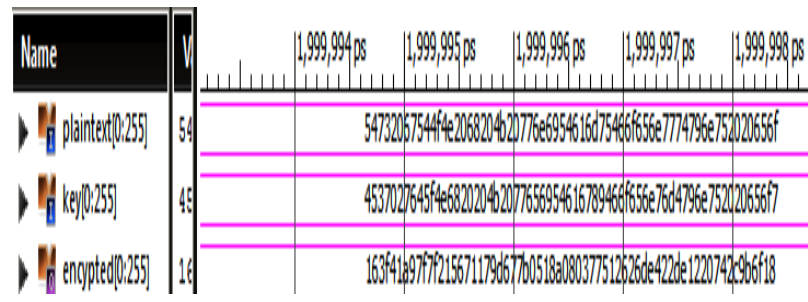

Fig 9: Simulation result of 256 bit

\subsection{Simulation Result of 512 Bit Plaintext And Key}

Plaintext:

013c6e4763eb9fa0ba84e81b430e093dc02f9392ab30afc775a4 8d40c65708f820cb2ba21f4e2274f48d067da9c0eb7f0e081b31 $54590 \mathrm{~b} 1 \mathrm{a} 7 \mathrm{a} 320 \mathrm{e} 5 \mathrm{~d} 62 \mathrm{c} 8 \mathrm{fe} 37$

Key:

7e1008493cdabbf2036fa968d43979c882feb32143554eb9cf89 d62f739f3141Aba332315e369f53fe8f19f613a15d81e1ec6998 654ff77ddc9a5c0eba19872e

Ciphertext: 


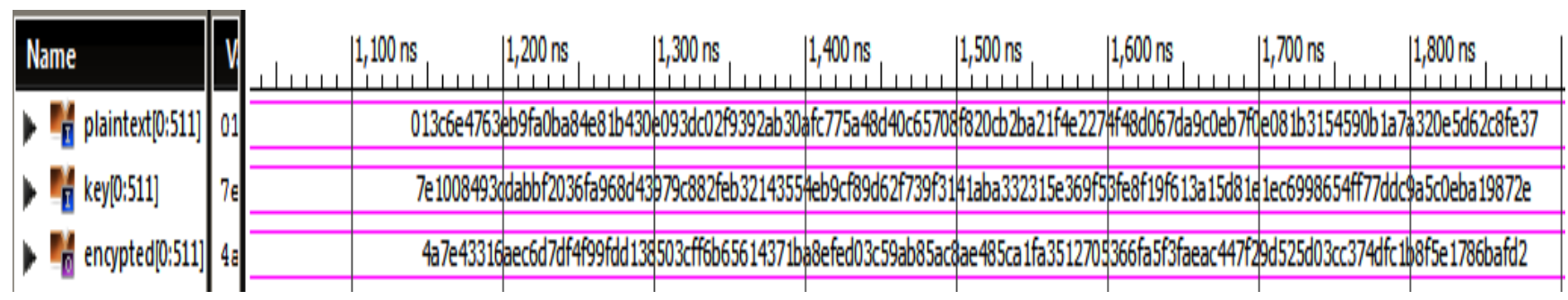

Fig 10: Simulation result of 512 bit

\begin{tabular}{|c|c|c|}
\hline Report & avigator & $\bar{x}$ \\
\hline View & & A \\
\hline & Default Activity Rates & \\
\hline & iummany & \\
\hline & Confidence Level & \\
\hline & Details & \\
\hline & By Hierarchy & \\
\hline & By Clock Domain & \\
\hline & 6. By Resource Type & $\equiv$ \\
\hline & Logic & \\
\hline & Signals & \\
\hline & Data & \\
\hline & $\boxminus$ Control & 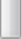 \\
\hline & Clock Enable & \\
\hline & Set/Reset & - \\
\hline Color & Source & \\
\hline & Estimated & \\
\hline & Default & \\
\hline & Calculated & \\
\hline
\end{tabular}

\begin{tabular}{|c|c|c|c|c|c|c|c|c|c|c|c|}
\hline A & B & $C D$ & $\mathrm{E}$ & $\mathrm{F}$ & G & H & $1 \mathrm{~J}$ & k & L & M & $\mathrm{N}$ \\
\hline Device & & On-Chip & Power (W) & \begin{tabular}{|l} 
Used \\
\end{tabular} & Avallable & Utitization ('\%) & Supply & Summay & Total & Dynamic & Quescoent \\
\hline Family & Vitex5 & Clocks & 0.075 & 195 & - & - & Source & Voltage & Cunrent (A) & Cunert (A) & Cument (A) \\
\hline Pat & xc5vkx50t & Logic & 0.000 & 17619 & 28800 & 61 & Vocint & 1.000 & 0.364 & \begin{tabular}{|l|l|}
0.075 \\
\end{tabular} & 0.288 \\
\hline Package & f11136 & Signals & 0.000 & 13175 & - & 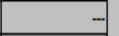 & Vocoulx & 2.500 & 0.062 & 0.000 & 0.062 \\
\hline$\overline{\text { Temp Grade }}$ & Commercial & $10 s$ & 0.000 & 11 & 480 & 0 & $\sqrt{100025}$ & 2.500 & 0.002 & 0.000 & 0.002 \\
\hline Process & Typical & Leakage & 0.449 & & & & & & & & \\
\hline$\overline{\text { Speed Grade }}$ & -1 & Total & 0.524 & & & & \multirow{2}{*}{\multicolumn{2}{|c|}{ Supply Power (W) }} & Total & Dynamic & \begin{tabular}{|l|} 
Quiescent \\
\end{tabular} \\
\hline & & & & & & & & & 0.524 & 0.075 & 0.449 \\
\hline Environment & & \multirow{3}{*}{\multicolumn{2}{|c|}{ Themal Properties }} & Effective TJA & Max Ambient. & Junction Temp & & & & & \\
\hline Ambient Temp (C) & 50.0 & & & $(\mathrm{C} N)^{2}$ & (C) & (c) & & & & & \\
\hline Use custom TJA? & No & & & 1.5 & 84.2 & 50.8 & & & & & \\
\hline Custom TJA (CM) & $\mathrm{NA}$ & & & & & & & & & & \\
\hline Ainflow (LFM) & 250 & & & & & & & & & & \\
\hline Heat Sink & Medium Profile & & & & & & & & & & \\
\hline Custom TSA (CM) & NA & & & & & & & & & & \\
\hline Board Selection & Medium $\left(10^{\prime \prime} \times 10^{\prime \prime}\right)$ - & & & & & & & & & & \\
\hline \# of Board Lajers & 8 to 11 & & & & & & & & & & \\
\hline Custom TJB (CNM) & $\mathrm{NA}$ & & & & & & & & & & \\
\hline Board Temperature (C & & & & & & & & & & & \\
\hline
\end{tabular}

Fig 11: Power Analysis of 192 bit

\begin{tabular}{|c|c|c|}
\hline \multicolumn{3}{|c|}{ Report Navigator } \\
\hline \multicolumn{2}{|l|}{ View } & $\wedge$ \\
\hline \multicolumn{3}{|c|}{$\begin{array}{l}\text { Default Activity Rates } \\
\text { Summary }\end{array}$} \\
\hline \multicolumn{3}{|c|}{ Summary } \\
\hline & Confidence Level & \\
\hline \multicolumn{3}{|c|}{ Details } \\
\hline \multicolumn{3}{|c|}{ By Hierarchy } \\
\hline \multicolumn{3}{|c|}{ By Clock Domain } \\
\hline \multicolumn{3}{|c|}{ 曰. By Resource Type } \\
\hline & Logic & \\
\hline \multicolumn{3}{|c|}{ Signals } \\
\hline \multicolumn{3}{|c|}{ Data } \\
\hline \multicolumn{3}{|c|}{ G Control } \\
\hline \multicolumn{3}{|c|}{ Clock Enable } \\
\hline & Set/Reset & + \\
\hline \multicolumn{3}{|c|}{ Source } \\
\hline L & \multicolumn{2}{|l|}{ Estimated } \\
\hline & \multicolumn{2}{|l|}{ Default } \\
\hline & \multicolumn{2}{|l|}{ Calculated } \\
\hline
\end{tabular}

\begin{tabular}{|c|c|c|c|c|c|c|c|c|c|c|c|}
\hline A & B & $C D$ & $\mathrm{E}$ & $\mathrm{F}$ & G & H & $\mathrm{I} \mathrm{J}$ & K & L & M & $\mathrm{N}$ \\
\hline Device & & On-Chip & Power (W) & Used & Avaliable & Utilization (\%) & Supply & Summary & Total & Dynamic & Quiescent \\
\hline Family & Vitex 5 & Clocks & 0.078 & 259 & - & - & Source & \begin{tabular}{|l|l} 
Voltage \\
\end{tabular} & Cunent $(A)$ & Cunent $(A)$ & Cunent (A) \\
\hline Pat & xc5uk50t & Logic & 0.000 & 27336 & 28800 & 95 & Vocint & 1.000 & 0.366 & \begin{tabular}{|l|}
0.078 \\
\end{tabular} & \begin{tabular}{|l|}
0.289 \\
\end{tabular} \\
\hline Package & $f 1136$ & Signals & 0.000 & 20107 & - & - & Vecaulx & 2.500 & 0.062 & 0.000 & 0.062 \\
\hline Temp Grade & Commercial & $10 s$ & 0.000 & & 480 & 0 & Vocoo25 & 2.500 & 0.002 & 0.000 & 0.002 \\
\hline Process & Typioal & Leakage & 0.449 & & & & & & & & \\
\hline Speed Grade & -1 & Total & 0.526 & & & & \multirow{2}{*}{\multicolumn{2}{|c|}{ Supply Power (W) }} & Total & Dynamic & Quiescent \\
\hline & & & & & & & & & 0.526 & $\begin{array}{l}0.078 \\
\end{array}$ & \begin{tabular}{|l|}
0.449 \\
\end{tabular} \\
\hline Environment & & \multirow{3}{*}{\multicolumn{2}{|c|}{ Themal Properties }} & Effective TJA & Max Ambient & Junction Temp & & & & & \\
\hline Ambient Temp (C) & 50.0 & & & $(\mathrm{CN})$ & (C) & (C) & & & & & \\
\hline Use custom TJA? & No & & & 1.5 & 84.2 & 50.8 & & & & & \\
\hline Custom TJA (CN) & NA & & & & & & & & & & \\
\hline Airflow (LFM) & 250 & & & & & & & & & & \\
\hline Heat Sink & Medium Profile & & & & & & & & & & \\
\hline Custom TSA (CN) & NA & & & & & & & & & & \\
\hline Board Selection & Medium $\left(10 " x 0^{\prime \prime}\right)$ - & & & & & & & & & & \\
\hline \# of Board Layers & 8to 11 & & & & & & & & & & \\
\hline Custom TJB (CN) & NA & & & & & & & & & & \\
\hline Board Temperature (C & & & & & & & & & & & \\
\hline
\end{tabular}

Fig 12: Power Analysis of 256 bit 


\begin{tabular}{|c|c|c|}
\hline \multicolumn{3}{|c|}{ Report Navigator } \\
\hline \multicolumn{2}{|l|}{ View } & $\wedge$ \\
\hline \multicolumn{3}{|c|}{ Default Activity Rates } \\
\hline \multicolumn{2}{|c|}{ Summary } & \\
\hline \multicolumn{2}{|c|}{ Confidence Level } & \\
\hline \multirow{2}{*}{\multicolumn{2}{|c|}{ G. Details }} & \\
\hline & By Hierarchy & \\
\hline \multicolumn{2}{|r|}{ By Clock Domain } & \\
\hline \multicolumn{2}{|r|}{ 曰- By Resource Type } & $\equiv$ \\
\hline \multicolumn{2}{|r|}{ Logic } & \\
\hline \multicolumn{2}{|r|}{$\boxminus$ Signals } & \\
\hline \multicolumn{3}{|c|}{ Data } \\
\hline \multicolumn{3}{|c|}{$\boxminus$ Control } \\
\hline \multicolumn{3}{|c|}{ Clock Enable } \\
\hline & Set/Reset & - \\
\hline \multicolumn{3}{|c|}{ Source } \\
\hline \multicolumn{3}{|c|}{ Estimated } \\
\hline \multicolumn{3}{|c|}{ Default } \\
\hline & \multicolumn{2}{|l|}{ Calculated } \\
\hline
\end{tabular}
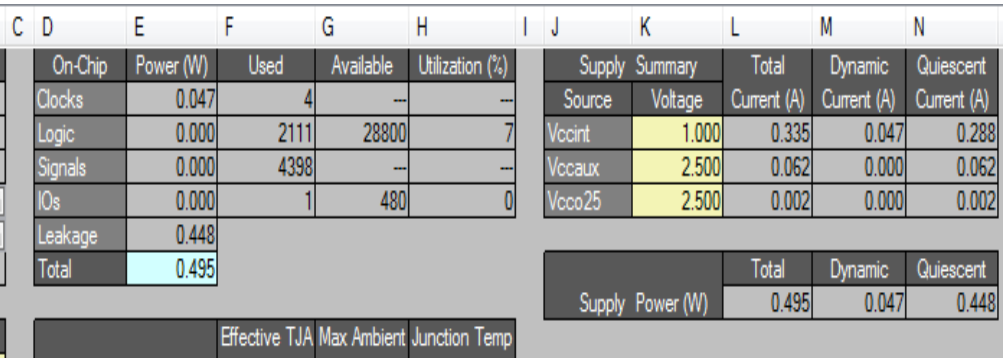

\section{Environment}

\begin{tabular}{l|l}
\hline Ambient Temp (C) & 50.0 \\
\hline
\end{tabular}

Use custom TJA?

Custom TJA (CN)

Aiflow (LFM)

Heat Sink

Custom TSA CNM

Board Selection

\# of Board Layers

Gustom TJ.J C CNW

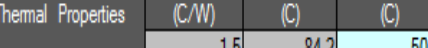

\begin{tabular}{|r|r|r|}
\hline 1.5 & 84.2 & 50.8 \\
\hline
\end{tabular}

Fig 13: Power Analysis of 512 bit

Table 1 Analysis of hardware required

\begin{tabular}{|l|c|c|c|l|l|l|l|l|l|}
\hline Operations & $\begin{array}{l}\text { Gates } \\
\mathbf{1 9 2}\end{array}$ & $\begin{array}{c}\text { Gates } \\
\mathbf{2 5 6}\end{array}$ & $\begin{array}{l}\text { Gates } \\
\mathbf{5 1 2}\end{array}$ & Garbage(192) & Garbage(256) & Garbage(512) & $\begin{array}{l}\text { Quantum } \\
\text { Cost(192) }\end{array}$ & $\begin{array}{l}\text { Quantum } \\
\text { Cost(256) }\end{array}$ & $\begin{array}{l}\text { Quantum } \\
\text { Cost(512) }\end{array}$ \\
\hline $\begin{array}{l}\text { Key } \\
\text { Expansion }\end{array}$ & 32 & 32 & 32 & 32 & 32 & 32 & 32 & 32 & 32 \\
\hline Add Round & 192 & 256 & 512 & 196 & 256 & 512 & 192 & 256 & 512 \\
\hline Shift Rows & 56 & 72 & 136 & 56 & 72 & 136 & 56 & 72 & 136 \\
\hline $\begin{array}{l}\text { Mixed } \\
\text { Column }\end{array}$ & 16 & 16 & 16 & 16 & 16 & 16 & 16 & 16 & 16 \\
\hline Total & 296 & 376 & 696 & 296 & 376 & 696 & 296 & 376 & 696 \\
\hline
\end{tabular}

Table 2 Power and Delay analysis

\begin{tabular}{|c|c|c|}
\hline Key Size & Delay(ns) & Power(watt) \\
\hline 192 & 34.522 & 0.524 \\
\hline 256 & 40.145 & 0.524 \\
\hline 512 & 74.922 & 0.495 \\
\hline
\end{tabular}

\section{CONCLUSION}

Attempt is made to increase the security level by using plain text size same as that of key size. And design is implemented using reversible logic gates to address the side channel power attacks in conventional AES. Design is verified using Xilinx tool and implemented on virtex5 board using chipscope. Power and delay is tabulated for 3 key size AES. It is found to be using lesser hardware as it is implemented using only Feynman gate whose quantum cost is 1 . Thus the increase in the message length as well as key w.r.t standard AES makes the AES algorithm more stronger against the new attacks and has an acceptable speed of data encryption and decryption.

\section{REFERENCES}

[1] Srinivas, NS Sai, and Md Akramuddin. "FPGA based hardware implementation of AES Rijndael algorithm for Encryption and Decryption." Electrical, Electronics, and Optimization Techniques (ICEEOT), International Conference on. IEEE, 2016.

[2] Nag, K. Sowmya, H. B. Bhuvaneswari, and A. C. Nuthan. "Implementation of advanced encryption Standard-192 bit using multiple keys." (2013): 2-26.

[3] Jonwal, Sheetal U., and Pratibha P. Shingare. "Advanced Encryption Standard (AES) implementation on FPGA 
with hardware in loop." Trends in Electronics and Informatics (ICEI), 2017 International Conference on. IEEE, 2017.

[4] Raj, Gaurav, Ram Charan Kesireddi, and Shruti Gupta. "Enhancement of security mechanism for confidential data using AES-128, 192 and 256bit encryption in cloud." Next Generation Computing Technologies (NGCT), 2015 1st International Conference on. IEEE, 2015.

[5] D'souza, Flevina Jonese, and Dakshata Panchal. "Advanced encryption standard (AES) security enhancement using hybrid approach." Computing, Communication and Automation (ICCCA), 2017 International Conference on. IEEE, 2017.

[6] Saicheur, Vatchara, and Krerk Piromsopa. "An implementation of AES-128 and AES-512 on Apple mobile processor." Electrical Engineering/Electronics, Computer, Telecommunications and Information Technology (ECTI-CON), 2017 14th International Conference on. IEEE, 2017.

[7] Moh'd, Abidalrahman, Yaser Jararweh, and Lo'ai Tawalbeh. "AES-512: 512-bit Advanced Encryption Standard algorithm design and evaluation." Information Assurance and Security (IAS), 2011 7th International Conference on. IEEE, 2011.
[8] Saberi, Iman, Bahareh Shojaie, and Mazleena Salleh. "Enhanced key expansion for AES-256 by using evenodd method." Research and Innovation in Information Systems (ICRIIS), 2011 International Conference on. IEEE, 2011

[9] H Rohini, Rajashekar,P Kumar: Design of basic sequential circuits using reversible logic. International conference on electrical, electronics, and optimization techniques(ICEEOT),March 2016, pp.2110-2115.

[10] Rohini H, Rajashekar S: Design of Reversible logic based combinational circuits. Communications on Applied Electronics (CAE),Sept 2016, Vol 5, pp. 38-43.

[11] Rohini S H, Jyoti R H, Rajashekar B. S: Reversible Logic Based Modified Design of AES-CBC Mode. Seventh International conference on Advanced electrica Measurement \& instrumentation Engineering (EMIE), 13,14 July 2018, pp.171-176.

[12] Rohini S. H, Nikhita M, Pooja A, Rajashekar B. S: Performance Analysis of AES-128bits,192bits \& 256bits using reversible logic. Seventh International conference on Advanced electrica Measurement \& instrumentation Engineering (EMIE), 13,14 July 2018, pp.165-170. 